NASA Technical Memorandum 106438

AIAA-93-2084

\title{
Blade Row Interaction Effects on Flutter and Forced Response
}

Daniel H. Buffum

Lewis Research Center

Cleveland, Ohio

(NASA-TM-106438) BLADE ROW INTERACTION EFFECTS ON FLUTTER AND

FORCED RESPONSE (NASA) $19 \mathrm{P}$
N94-21586

Unclas

G3/07 0198109

Prepared for the

29th Joint Propulsion Conference

cosponsored by the AIAA, SAE, ASME, and ASEE

Monterey, California, June 28-July 1, 1993 


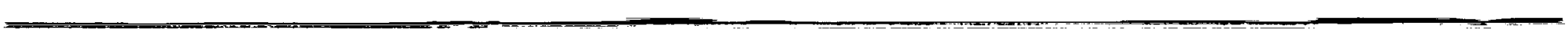

mand 


\title{
Blade Row Interaction Effects on Flutter and Forced Response
}

\author{
Daniel H. Buffum \\ NASA Lewis Research Center \\ Cleveland, Ohio
}

\section{Abstract}

In the flutter or forced response analysis of a turbomachine blade row, the blade row in question is commonly treated as if it is isolated from the neighboring blade rows. Disturbances created by vibrating blades are then free to propagate away from this blade row without being disturbed. In reality, neighboring blade rows will reflect some portion of this wave energy back toward the vibrating blades, causing additional unsteady forces on them. It is of fundamental importance to determine whether or not these reflected waves can have a significant effect on the aeroelastic stability or forced response of a blade row. Therefore, a procedure to calculate intra-blade-row unsteady aerodynamic interactions has been developed which relies upon results available from isolated blade row unsteady aerodynamic analyses. In addition, an unsteady aerodynamic influence coefficient technique is used to obtain a model for the vibratory response in which the neighboring blade rows are also flexible. The flutter analysis shows that interaction effects can be destabilizing, and the forced response analysis shows that interaction effects can result in a significant increase in the resonant response of a blade row,

\section{Nomenclature}

a speed of sound

$A \quad$ nondimensional acoustic wave coefficient

$c \quad$ chord

$G$ nondimensional gust coefficient

$i \quad \sqrt{-1}$

Im imaginary part

$M \quad$ relative Mach number

$R \quad$ nondimensional reflection coefficient

$\mathrm{Re}$ real part

$s$ blade tangential spacing

$T$ nondimensional transmission coefficient

Copyright 01993 by AIAA Inc. No copyright is asserted in the United States under Title 17, U.S. Code. The U.S. Government has a royalty-free license to exercise all rights under the copyright claimed herein for Governmental purposes. All other rights are reserved by the copyright owner.

$\begin{array}{ll}U & \text { rotor wheel speed } \\ V & \text { fluid velocity } \\ \alpha & \text { angle of blade oscillation } \\ \beta & \text { interblade phase angle } \\ \delta & \text { axial gap between neighboring blade rows } \\ \eta & \text { tangential coordinate } \\ \xi & \text { axial coordinate } \\ \rho & \text { fluid density } \\ \omega & \text { angular frequency } \\ \bar{\omega} & \text { reduced frequency, } \omega c / V\end{array}$

\section{Introduction}

In the flutter analysis of a turbomachine blade row, the blade row is commonly assumed to be isolated disturbances created by the vibrating blades are free to propagate away from the blade row without being disturbed. Thus any reflections of these outgoing waves by other structural members or nonuniformities in the mean flow field are neglected. Although the forced response problem is by definition concemed with blade row interaction (with response to inlet distortion an important exception), forced response analyses also typically neglect any reflections of outgoing waves. However, in an engine environment, structural elements such as neighboring blade rows or struts and nonuniformities in the mean flow field will generally reflect some of this wave energy back toward the vibrating blades, causing additional unsteady forces on them. Whether or not these reflected waves can significantly affect the aeroelastic stability or forced response of a blade row is a question of fundamental importance.

Several investigations have focused on the unsteady aerodynamic interaction between two rigid blade rows. Kaji and Okazaki (1970) investigated the unsteady aerodynamic interaction of two blade rows for the purpose of predicting rotor-stator interaction noise. They obtained a simultaneous solution to the unsteady lift distributions on both of the blade rows. Hanson (1992) modified Smith's (1972) isolated blade row unsteady aerodynamic analysis to predict rotor-stator interaction noise, essentially extending Kaji and Okazaki's work to include effects of frequency scattering and mean flow turning by the blade rows. For a counter-rotating propfan in incompressible flow, Chen and 
Williams (1991) used a panel method to determine the unsteady loads on rigid propeller blades. From the point-of-view of the present investigation, all of these investigations are somewhat limited because they did not delve into the aeroelastic problem and they were limited to two blade rows.

One investigation that did consider the aeroelastic effects of interactions of a vibrating blade row and an adjacent structure was that of Williams, Cho and Dalton (1991). A three-dimensional linearized compressible panel method was used to calculate the unsteady aerodynamics of a ducted fan. The aeroelastic analysis allowed flexibility of both the fan and the duct. The duct was found to have a destabilizing effect on the fan.

A number of time-accurate solutions to the Euler and Navier-Stokes equations for rotor-stator interaction have been presented, e.g., Rai (1987), Jorgensen and Chima (1989), Giles (1990). Due to the large computing time required to obtain solutions for just two blade rows, time-marching codes such as these are not practical for routine aeroelastic analysis purposes.

The purpose of this paper is to investigate the effects of blade row interaction on flutter and forced response. A model for interaction between any number of blade rows is proposed which relies upon unsteady aerodynamic coefficients obtained from isolated blade row unsteady aerodynamic analyses. This allows the use of computationally-efficient linearized analyses, which are currently favored for turbomachinery aeroelastic predictions, for the analysis of multiple blade row unsteady aerodynamic interactions.

The linearized analyses are generally capable of providing solutions for the unsteady blade forces and the outgoing waves generated by two classes of disturbances: (1) blade motion and (2) incoming waves such as acoustic and vorticity waves. Thus the unsteady aerodynamic coefficients which quantify the wave reflection and transmission characteristics of the (rigid) blade rows, as required to aerodynamically couple the blade rows, are provided by the linearized unsteady aerodynamic analyses. In addition, the response coefficients due to blade motion allow the calculation of influence coefficients which express the unsteady forces on one blade row due to the motion of another. By combining the various influence coefficients with equations of motion for each of the blade rows, a system of equations may be obtained which models what will be referred to as the 'dynamic coupling' of flexible blade rows.

In this paper, a model for unsteady aerodynamic interaction between any number of blade rows is developed. The coupled equations of motion for the flexible blade rows, which rely upon unsteady aerodynamic influence coefficients for the intra-blade row coupling, are derived. A system of linear equations is found for the unsteady aerodynamic influence coefficients. Whitehead's subsonic flat plate cascade analysis, LINSUB (Whitehead, 1987), is used to provide the unsteady aerodynamic input for the influence coefficient solution although this model is suitable for use with the predictions of more sophisticated isolated blade row analyses which account for nonuniform mean flow fields (e.g., Verdon (1990), Hall and Clark (1991), Fang and Atassi (1993)). Before applying this technique to aeroelastic problems, the predictions of the unsteady aerodynamic model are compared with those of Kaji and Okazaki for outgoing acoustic wave amplitudes due to rotor-stator interaction. Then the model is used to investigate the effects of blade row interaction on the flutter and forced response of systems of three blade rows.

\section{Analysis}

\subsection{Equation of motion}

A single degree-of-freedom model for torsional oscillation of an isolated blade row will be used to account for the effect of blade row interaction on the vibratory response of a row of identical blades. From Chiang (1988), the equation of motion for the small amplitude torsional oscillation of a two-dimensional section of a reference blade may be expressed as

$$
I \frac{d^{2} \bar{\alpha}}{d t^{2}}+(1+2 i g) l \omega_{u}^{2} \bar{\alpha}=\bar{M}
$$

$\bar{\alpha}=\bar{\alpha}(t)$ is the angle of oscillation, $I$ is the mass moment of inertia about the elastic axis, $g$ is the structural damping coefficient for torsion and $\omega_{u}$ is the undamped natural frequency for this mode of oscillation. $\bar{M}$ is the unsteady aerodynamic moment per unit span about the elastic axis.

Simple harmonic motion at frequency $\omega$ and a fixed interblade phase angle is assumed. Let the oscillation angle for a reference blade be $\bar{\alpha}(t) \equiv \alpha e^{i \omega t}$ and the unsteady aerodynamic moment be $\bar{M}(t)=\widetilde{M} e^{i \omega t}$. Substituting these into Equation 1 and differentiating gives

$$
\left[-I \omega^{2}+(1+2 i g) I \omega_{u}^{2}\right] \alpha=\tilde{M}
$$

For the purpose of calculating $\tilde{M}$, the unsteady aerodynamic disturbances are assumed to be small relative to an inviscid, subsonic mean flow, resulting in a linear problem for the first order unsteady disturbance field. In this investigation, the unsteady disturbances are limited to acoustic and vorticity waves. Because the unsteady disturbance field is linear, the unsteady aerodynamic moment $\tilde{M}$ may be determined by the superposition of motion-dependent and motion-independent parts. Typically, these moments are calculated under the 
assumption that the blade row whose dynamic response is being analyzed is isolated from any other blade rows. Consider, for example, the response of a stator embedded in a multistage turbomachine to the wakes of an upstream rotor. The unsteady aerodynamic coefficients are calculated based on a wake profile specified at the stator leading edge locus and the tangential velocity of the rotor. As a result of the unsteady interaction of the wakes with the stator, waves are created which propagate away from the stator: acoustic waves propagate both upstream and downstream, and vorticity waves convect downstream. If the stator is treated as an isolated blade row, as shown schematically in Figure 1 , these waves are free to propagate away from the stator undisturbed. In reality, these waves will impact neighboring blade rows, Figure 2. Even if the neighboring blade rows are perfectly rigid, they will emit additional acoustic and vorticity waves in response to the waves from the stator, and these waves, in turn, may further load the stator, which then emits more waves in response, and so on. Through these waves the blade rows are aerodynamically coupled. The question is, then, does this aerodynamic coupling between the blade rows have a significant effect on the aeroelastic response of the stator?

One may carry this a step further and consider the vibratory response of the neighboring blade rows to the waves initiated by the stator/wake interaction. By allowing all of the blades to be flexible, they will respond by vibrating and emitting additional waves which will depend upon their vibrations. These motion-generated waves will further load the stator and result in additional waves ad infinitum. By allowing all of the blade rows to be flexible, the blade rows become dynamically coupled. Thus a second related question is, can the dynamic coupling of the blade rows significantly affect the vibratory response of the primary row?

Since the unsteady aerodynamic problem has been assumed to be governed by a system of linear equations, the aerodynamic blade row interactions can be expressed as nondimensional aerodynamic influence coefficients. Let $\mathrm{C}_{j k}$ be the nondimensional moment coefficient for the $j$-th blade row due to oscillation of the $k$-th blade row which includes effects due to wave interaction (i.e., reflection and transmission of acoustic waves, shed vorticity) with the other non-vibrating blade rows. Thus $C_{i j}$ is the moment coefficient on the $j$-th blade row due to its own oscillations. These coefficients can be used to dynamically couple any number of blade rows. For a system of $n$ blade rows restricted to torsional oscillation, Equation 2 will be applied to each blade row. The unsteady aerodynamic influence coefficients are used to express the moment in Equation 2 as the linear combination of the influences of all of the blade rows. Assuming that the forced response of the $j$-th blade row is being investigated, the unsteady aerodynamic moment on a reference blade of that row is

$$
\begin{aligned}
\tilde{M}_{j}= & \rho_{j} V_{j}^{2} c_{j}^{2}\left(C_{j 1} \alpha_{1}+C_{j 2} \alpha_{2}+\ldots+C_{j j} \alpha_{j}+\ldots+C_{j n} \alpha_{n}+\right. \\
& {\left.[C G]_{j 1} \frac{w_{1}}{\nu_{1}}+[C G]_{j 2} \frac{w_{2}}{V_{2}}+\ldots+[C G]_{j j} \frac{w_{j}}{\nu_{j}}+\ldots+[C G]_{j n} \frac{w_{n}}{V_{n}}\right) . }
\end{aligned}
$$

$\rho_{j}$ is the gas density, $V_{j}$ is the relative inlet velocity and $c_{j}$ is the chord for blade row $j . \alpha_{k}$ is the complex-valued angle of oscillation of the reference blade of row $k$. [CG $]_{j k}$ is the nondimensional moment coefficient for row $j$ due to a gust acting on row $k$ with upwash $w_{k}$-typically $[C G]_{j k}$ is used to model the effects of viscous wakes. Substituting Equation 3 into Equation 2 then rearranging into a nondimensional form results in

$$
\begin{aligned}
& C_{j 1} \alpha_{1}+C_{j 2} \alpha_{2}+\ldots \\
& +\left[\frac{\pi}{16} \bar{\omega}_{j}^{2} r_{j}^{2} \mu_{j}\left(1-\left(1+2 i g_{j}\right) \frac{\omega_{u j}^{2}}{\omega_{j}^{2}}\right)+C_{i j}\right] \alpha_{j}+ \\
& \ldots+\mathrm{C}_{\lambda(n-1)} \alpha_{n-1}+\mathrm{C}_{j n} \alpha_{n}=-\sum_{k=1}^{n} \frac{w_{k}}{V_{k}}[\mathbf{C G}]_{j k}
\end{aligned}
$$

where $\bar{\omega}_{j}=\frac{\omega_{j} c_{j}}{V_{j}}$ is the reduced frequency, $r_{j}=\sqrt{\frac{I_{j}}{m_{j}\left(c_{j} / 2\right)^{2}}}$ is the nondimensional radius of gyration of the blade section and $\mu_{j}=\frac{m_{j}}{\pi \rho\left(c_{j} / 2\right)^{2}}$ is the mass ratio of that section. Letting

$$
\mathrm{S}_{j}=\frac{\pi}{16} \bar{\omega}_{j}^{2} r_{j}^{2} \mu_{j}\left[1-\left(1+2 i g_{j}\right) \gamma_{j}\right]
$$

where $\gamma_{j}=\omega_{u j}^{2} / \omega_{j}^{2}$, Equation 4 becomes

$$
\mathbf{C}_{j 1} \alpha_{1}+\ldots+\left(\mathbf{S}_{j}+\mathbf{C}_{j j}\right) \alpha_{j}+\ldots+\mathbf{C}_{j n} \alpha_{n}=-\sum_{k=1}^{n} \frac{w_{k}}{V_{k}}[\mathbf{C G}]_{j k}
$$

The only relevant values of $[\mathrm{CG}]_{j k}$ are those with the frequency $\omega_{j}$. In many cases, the gusts acting at any row $k \neq j$ will not result in excitation of row $j$ at $\omega_{j}$, hence $[\mathrm{CG}]_{j k}=0$ for $k \neq j$ and Equation 5 becomes

$$
\mathbf{C}_{j 1} \alpha_{1}+\ldots+\left(\mathbf{S}_{j}+\mathbf{C}_{j i}\right) \alpha_{j}+\ldots+\mathbf{C}_{j n} \alpha_{n}=-\frac{w_{j}}{V}[\mathbf{C G}]_{j j} .
$$

In this case, the equation of motion for a blade row $k \neq j$ is

$$
\mathbf{C}_{k 1} \alpha_{1}+\ldots+\left(\mathbf{S}_{k}+\mathbf{C}_{k k}\right) \alpha_{k}+\ldots+\mathbf{C}_{k n} \alpha_{n}=-\frac{w_{j}}{V_{j}}[\mathbf{C G}]_{k j} \text {. }
$$

Using Eq. 6a for blade row $j$ and Eq. $6 \mathrm{~b}$ for the remaining $n-1$ blade rows, a linear system of equations is obtained which may be solved for the dynamics of these blade rows due to a gust acting on row $j$. This system may be expressed as

$$
\mathbf{A x}=\mathbf{B}
$$

where 


$$
\begin{gathered}
\mathbf{A}=\left[\begin{array}{cccccc}
\mathbf{D}_{1} & \mathbf{C}_{12} & & \ldots & \mathbf{C}_{1(n-1)} & \mathbf{C}_{1 n} \\
\mathbf{C}_{21} & \mathbf{D}_{2} & & \ldots & \mathbf{C}_{2(n-1)} & \mathbf{C}_{2 n} \\
\vdots & \vdots & \ddots & & & \vdots \\
\mathbf{C}_{j 1} & \mathbf{C}_{j 2} & \ldots & \mathbf{D}_{j} & \mathbf{C}_{j(n-1)} & \mathbf{C}_{j n} \\
\vdots & \vdots & & \ddots & \vdots & \vdots \\
\mathbf{C}_{(n-1) 1} & \mathbf{C}_{(n-1) 2} & \ldots & & \mathbf{D}_{n-1} & \mathbf{C}_{(n-1) n} \\
\mathbf{C}_{n 1} & \mathbf{C}_{n 2} & & \ldots & \mathbf{C}_{n(n-1)} & \mathbf{D}_{n}
\end{array}\right] \\
\multicolumn{5}{c}{\mathbf{D}_{j}=\mathbf{S}_{j}+\mathbf{C}_{j j}} \\
\mathbf{x}=\left[\alpha_{1}, \alpha_{2}, \ldots, \alpha_{j}, \ldots, \alpha_{n-1}, \alpha_{n}\right]^{\mathrm{T}}
\end{gathered}
$$

and

$$
\begin{array}{r}
\mathbf{B}=-\left[\frac{w_{j}}{v_{j}}[\mathbf{C G}]_{1 j}, \frac{w_{j}}{V_{j}}[\mathbf{C G}]_{2 j}, \ldots, \frac{w_{j}}{V_{j}}[\mathbf{C G}]_{j j}, \ldots,\right. \\
\left.\frac{w_{j}}{V_{j}}[\mathbf{C G}]_{(n-1) j}, \frac{w_{j}}{V_{j}}[\mathbf{C G}]_{n j}\right]^{\mathrm{T}}
\end{array}
$$

Specifying $B=0$ in Equation 7 results in a homogeneous system appropriate for determination of the system stability:

$$
\mathbf{A x}=0
$$

A meaningful solution exists only if the determinant of the coefficient matrix $\mathbf{A}$ is equal to zero.

For any blade row $k$, the structural coefficients $r_{k}, \mu_{k}$ and $g_{k}$ are assumed to be known. To investigate the stability of a particular row, row $j, \bar{\omega}_{j}$ is chosen and this, along with the geometry and mean flow field, sets the values of $\bar{\omega}_{k}$ for the other blade rows (see Appendix B). The frequency ratios $\gamma_{k}$ are unknown, but they may be expressed as a functions of $\gamma_{j}$ :

$$
\begin{aligned}
\gamma_{k} & =\frac{\omega_{u k}^{2}}{\omega_{u j}^{2}} \frac{\omega_{u j}^{2}}{\omega_{j}^{2}} \frac{\omega_{j}^{2}}{\omega_{k}^{2}} \\
& =\frac{\omega_{u k}^{2}}{\omega_{u j}^{2}} \gamma_{j} \frac{\omega_{j}^{2}}{\omega_{k}^{2}} .
\end{aligned}
$$

$\omega_{u k}$ and $\omega_{u j}$ are assumed to be known, and the ratios $\omega_{j} / \omega_{k}$ will also be known due to their dependence on known parameters. Thus the determinant of the coefficient matrix yields a polynomial of degree $n$ in $\gamma_{j}$ which may be solved for $\gamma_{j}$.

The system stability is determined by the values of $\gamma_{j}$. Since

$$
\frac{i \omega_{j}}{\omega_{u j}}=\frac{i}{\sqrt{\gamma_{j}}}
$$

and $\overline{\alpha_{j}}(t)=\alpha_{j} e^{i \omega / t}, \quad \operatorname{Re}\left(i / \sqrt{\gamma_{j}}\right)>0$ indicates growing oscillations for blade row $j$ and blade rows $k$ (through the dependence of $\omega_{k}$ on $\omega_{j}$ ) and therefore system instability or flutter. $\operatorname{Im}\left(i / \sqrt{\gamma_{j}}\right)$ is the ratio of the blade row $j$ oscillation frequency to its natural frequency.

\subsection{Coupled blade row unsteady aerodynamics}

Expressions for the unsteady aerodynamic influence coefficients will now be developed. The mean flow field is assumed to be subsonic, and only those acoustic waves which propagate unattenuated will be considered. Blade row geometry definitions and flow field definitions are shown in Figure 3.

Assume that blade row $j$ is oscillating harmonically with unit amplitude at frequency $\omega_{j}$ and interblade phase angle $\beta_{j}$. Due to these oscillations, acoustic waves propagate both upstream and downstream, and vorticity waves are convected downstream. As shown in Appendix A, acoustic waves will propagate unattenuated through a uniform flow field of Mach number $M<1$ only if the tangential wavenumber $m$ (i.e., the $\eta$ component of the wavenumber) satisfies

$$
m>\frac{\omega}{a}\left(\frac{M_{\mathfrak{\eta}} \pm \sqrt{1-M_{\xi}^{2}}}{1-M^{2}}\right) .
$$

$M_{\xi}$ and $M_{\eta}$ are the $\xi$ and $\eta$ components of the Mach number, $\omega$ is the frequency and $a$ is the speed of sound. If this condition is met, two propagating acoustic waves will be produced with tangential wavenumber $m$, one propagating upstream and the other downstream. The (-) sign in Equation 14 corresponds to the upstream-nunning wave and + corresponds to the downstream-running wave; the $<$ symbol corresponds to the + and the $>$ corresponds to the $(-)$. These waves are said to be superresonant or cut-on. Otherwise, the waves decay exponentially with axial distance and are said to be subresonant or cut-off. To be consistent with the interblade phase angle $\beta_{j}, m$ must satisfy

$$
m s_{j}=\beta_{j}+2 \pi r, \quad r=0, \pm 1, \pm 2, \ldots
$$

where $S_{j}$ is the spacing of the row $j$ blades. As shown in the Appendix, scattering of these waves at the other blade rows results in waves returning to row $j$ which are generally not at the frequency of interest. Although these waves might eventually be scattered back to the frequency of interest, the scattered waves will be neglected.

Let the waves generated by the motion of row $j$ be denoted $G_{M, j}$ for the gust or vorticity wave, $A_{M, j}$ for the upstream-traveling acoustic wave and $A_{M, j}^{+}$for the downstream-traveling acoustic wave. Superscripts of $(-)$ and $(+)$ are used to indicate the directions of acoustic waves, $(-)$ being a wave going toward $-\infty$ (upstream) and $(+)$ being toward $+\infty$ (downstream). To avoid unnecessary 
complications in the present explanation, acoustic waves are assumed to propagate unattenuated in both the upstream and downstream directions for only one tangential wavenumber consistent with the interblade phase angle. Assuming that a dynamic steady state is reached for all the resulting waves, each blade row $k$ will have the net effects of three different incoming waves acting upon it, a gust, $G_{l, k}$, an upstream-running acoustic wave, $A_{j, k}^{-}$, and a downstream-running acoustic wave, $A_{1, k}^{+}$. The outgoing waves at row $k$ are linearly related to the incoming waves by the various reflection and transmission coefficients of the blade row. For example, the outgoing upstream-running acoustic wave will be

$$
A_{O, k}^{-}=A_{I, k}^{-} T_{k}^{-}+A_{l, k}^{+} R_{k}^{+}+G_{I, k} A_{G, k}^{-}
$$

where, for row $k, T_{k}$ is the transmission coefficient for upstream-traveling acoustic waves, $R_{k}^{+}$is the reflection coefficient for downstream-traveling acoustic waves and $A_{G, k}^{-}$is the upstream-running acoustic wave due to an incoming gust. Similarly, the other two outgoing waves are a linear combination of the incoming waves:

$$
A_{O, k}^{+}=A_{I, k}^{-} R_{k}^{-}+A_{l, k}^{+} T_{k}^{+}+G_{l, k} A_{G, k}^{+}
$$

and

$$
G_{O, k}=A_{l, k}^{-} G_{A, k}^{-}+A_{l, k}^{+} G_{A, k}^{+}+G_{l, k} G_{G, k} .
$$

The new coefficients $G_{A, k}^{-}, G_{A, k}^{+}$and $G_{G, k}$ are the resulting gusts or vorticity waves due to an upstream-running acoustic wave, a downstream-running acoustic wave and a gust, respectively, incident on row $k$.

By defining the outgoing and incoming waves at common interfaces between the blade rows, the outgoing waves at row $k$ are related to the incoming waves at adjacent blade rows by

$$
\begin{aligned}
& A_{O, k}^{-}=A_{l, k-1}^{-} \\
& A_{O, k}^{+}=A_{l, k+1}^{+} \\
& G_{O, k}=G_{I, k+1}
\end{aligned}
$$

so that Equations 17 through 19 become

$$
\begin{gathered}
A_{I, k-1}^{-}=A_{I, k}^{-} T_{k}+A_{I, k}^{+} R_{k}^{+}+G_{I, k} A_{G, k}^{-} \\
A_{I, k+1}^{+}=A_{I, k}^{-} R_{k}^{-}+A_{I, k}^{+} T_{k}^{+}+G_{I, k} A_{G, k}^{+} \\
G_{I, k+1}=A_{I, k}^{-} G_{A, k}^{-}+A_{I, k}^{+} G_{A, k}^{+}+G_{I, k} G_{G, k} .
\end{gathered}
$$

For the blade rows neighboring the oscillating row, the incoming waves may be split into known parts due to the oscillation and unknown parts, indicated by hats, due to the ensuing interactions with the other blade rows:

$$
\begin{aligned}
& A_{I, j-1}^{-}=\hat{A}_{I, j-1}^{-}+A_{M, j}^{-} \\
& A_{I, j+1}^{+}=\hat{A}_{I, j+1}^{+}+A_{M, j}^{+} \\
& G_{I, j+1}=\hat{G}_{I, j+1}+G_{M, j}
\end{aligned}
$$

Then, for row $j$ and its immediate neighbors, the system of equations is, with all of the unknowns collected on the right hand side and the known terms due to the motion of row $j$ on the left hand side,

$$
\begin{gathered}
A_{M, j}^{-}=-\hat{A}_{I, j-1}^{-}+A_{I, j}^{-} T_{j}^{-}+A_{I, j}^{+} R_{j}^{+}+G_{I, j} A_{G, j}^{-} \\
0=-A_{I, j-1}^{+}+A_{I, j-2}^{-} R_{j-2}^{-}+A_{I, j-2}^{+} T_{j-2}^{+}+G_{I, j-2} A_{G, j-2}^{+} \\
0=-G_{I, j-1}+A_{I, j-2}^{-} G_{A, j-2}^{-}+A_{I, j-2}^{+} G_{A, j-2}^{+}+G_{I, j-2} G_{G, j-2} \\
-A_{M, j}^{+} R_{j+1}^{+}-G_{M, j} A_{G, j+1}^{-}=-A_{I, j}^{-}+A_{I, j+1}^{-} T_{j+1}^{-}+ \\
\hat{A}_{I, j+1}^{+} R_{j+1}^{+}+\hat{G}_{l, j+1} A_{G, j+1}^{-} \\
-A_{M, j}^{-} R_{j-1}^{-}=-A_{I, j}^{+}+\hat{A}_{I, j-1}^{-} R_{j-1}^{-}+A_{I, j-1}^{+} T_{j-1}^{+}+G_{I, j-1} A_{G, j-1}^{+}(23) \\
-A_{M, j}^{-} G_{A, j-1}^{-}=-G_{I, j}+\hat{A}_{I, j-1}^{-} G_{A, j-1}^{-}+A_{I, j-1}^{+} G_{A, j-1}^{+}+ \\
G_{I, j-1} G_{G, j-1}
\end{gathered}
$$$$
0=-A_{I, j+1}^{-}+A_{I_{, j+2}}^{-} T_{j+2}^{-}+A_{J, j+2}^{+} R_{j+2}^{+}+G_{I, j+2} A_{G, j+2}^{-}
$$$$
A_{M, j}^{+}=-\hat{A}_{I, j+1}^{+}+A_{l, j}^{-} R_{j}^{-}+A_{I, j}^{+} T_{j}^{+}+G_{l, j} A_{G, j}^{+}
$$$$
G_{M, j}=-\hat{G}_{I, j+1}+A_{l, j}^{-} G_{A, j}^{-}+A_{l, j}^{+} G_{A, j}^{+}+G_{I, j} G_{G, j}
$$

Assuming that three blade rows will be analyzed, the present system of 9 equations has 15 unknowns because the incoming waves to rows $(j-2)$ and $(j+2)$ are required. By assuming that no waves internal to the system are reflected by these two rows, $R_{j-2}^{-}=G_{A, j-2}^{-}=R_{j+2}^{+}=A_{G, j+2}^{-}=0$, and that there are no waves incoming from sources external to the system, $A_{j, j-2}^{+}=G_{I, j-2}=A_{1, j+2}^{-}=0$, the number of unknowns is reduced to 9 . Thus the system of equations may be solved for the incoming waves to each of the three blade rows. Using coefficients provided by the isolated blade row analysis which relate the moment on a blade to the incoming waves, the unsteady moment on each blade row $k$ due to oscillation of row $j$ - the influence coefficient $C_{k j}$ - may then be determined. Similarly, by using the waves generated by a unit amplitude gust acting on row $j$ as the known terms in Equations 23, the gust coefficients $[\mathbf{C G}]_{k j}$ may also be determined.

In this investigation, Whitehead's (1987) linearized subsonic flat plate cascade code, LINSUB, which is based on Smith (1972), will be used to supply the required aerodynamic quantities. It is based on assumptions of inviscid, isentropic, subsonic flow through an isolated, 
infinite cascade of equally-spaced flat plate airfoils. The airfoils are set at zero mean incidence so that the mean flow is uniform, and the unsteady disturbances created by the airfoil oscillations or incoming gusts are assumed to create small unsteady disturbances to the mean flow, resulting in a linear problem for the unsteady flow. LINSUB calculates moment coefficients due to oscillation of the cascade or due to incoming acoustic or vorticity gusts, and outgoing acoustic and vorticity waves due to oscillation of the cascade or due to incoming waves. Thus all the coefficients required by Equation 23 can be obtained from LINSUB.

\section{Results}

This model for unsteady aerodynamic blade row interaction will be applied to both the flutter and forced response of the middle blade row in a system of three blade rows. Before investigating these aeroelastic problems, the predictions of the unsteady aerodynamic model will be compared with the predictions of Kaji and Okazaki (1970) for the outgoing acoustic wave amplitudes due to rotor-stator interaction.

\subsection{Acoustic wave generation by rotor-stator interaction}

Kaji and Okazaki investigated the unsteady aerodynamic interaction of two rigid blade rows in motion relative to one another for the purpose of predicting rotor-stator interaction noise. The mean flow was a uniform, compressible stream about two cascades of flat plates aligned with the flow. They obtained a simultaneous solution to the unsteady lift distributions on both of the blade rows and determined the resulting outgoing acoustic waves. For excitation of the downstream blade row by wakes from the upstream row, the wake model of Silverstein, Katzoff and Bullivant (1939) was used to specify the wake velocity distribution at the leading edge of the downstream blade row.

The stagger angles of the blade rows are $\gamma_{1}=-\gamma_{2}=30^{\circ}$, giving identical relative Mach numbers for the two rows. The solidity of each row is 1 and the reduced frequency is $\pi$. Comparison between the predictions of the present method and those of Kaji and Okazaki is shown in Figure 4. The amplitudes of the first harmonic upstream and downstream acoustic waves are shown as a function of the relative Mach number into the blade rows and the axial gap between the blade rows as a fraction of chord. There is generally good agreement between the predictions for both of the axial gaps.

\subsection{Flutter}

To investigate the effects of unsteady aerodynamic blade row interaction on flutter, two configurations of three blade rows were analyzed. The first configuration consists of a staggered rotor placed between unstaggered stators while the second has an unstaggered stator between staggered rotors. To help isolate various effects, the stability of the middle blade row will be determined for three different cases: (1) the middle row is treated as if it is an isolated blade row, so there is no unsteady aerodynamic coupling between the blade rows (the 'NC' solution); (2) unsteady aerodynamic coupling occurs between the middle row and its neighbors, but the neighboring blade rows are rigid (the 'AC' solution); (3) unsteady aerodynamic coupling occurs and the secondary blade rows are now flexible, hence the blade rows are dynamically coupled (the 'DC' solution). Variation of the blade row spacing as a technique for enhancing blade row stability will be investigated.

System parameters for configuration I are shown in Table 1. The stators are unstaggered and the axial Mach number relative to the stators is 0.4 . Because the relative flow is required to be aligned with the rotor and the rotor is staggered $60^{\circ}$, the rotor relative Mach number is 0.8 . Rotor velocity triangles were then used to find the rotor blade Mach number, $U / a=-0.693$. For the rotor geometry, mean flow conditions and reduced frequency $\bar{\omega}_{2}=1$, the acoustic resonances occur at $\beta_{2}=-20.3^{\circ}$ and $\beta_{2}=146.4^{\circ}$. The radii of gyration and mass ratios are similar to those used by Chiang (1988), and are believed to be reasonable values for titanium compressor blades. For the initial calculations, the leading edge loci of the blade rows are at axial locations $-1.0,0.1$ and 1.5 chord, so there is a 0.1 chord axial gap between the rotor and the upstream stator and a 0.9 chord axial gap between the rotor and the downstream stator. 
Table 1 Blade row configuration I

\begin{tabular}{|c|c|c|c|}
\hline ४৮. & Stator 1 & Rotor & Stator 2 \\
\hline Blade row index, $k$ & 1 & 2 & 3 \\
\hline Chord, $c$ ? & 1 & 1 & 1 \\
\hline Solidity, $c / s$ & 1.5 & 1.4 & 1.5 \\
\hline Stagger angle (deg.) & 0 & 60 & 0 \\
\hline Relative Mach no., $M$ & 0.4 & 0.8 & 0.4 \\
\hline Blade Mach number, $U \%$ & 0 & -0.693 & 0 \\
\hline Elastic axis / chord & 0.4 & 0.4 & 0.4 \\
\hline Radius of gyration & 0.4 & 0.4 & 0.4 \\
\hline Mass ratio & 200 & 200 & 200 \\
\hline Natural freq, $\omega_{\nu} / \omega_{\nu \text { Roor }}$ & 1 & 1 & 1 \\
\hline Damping coefficient & $0.5 \%$ & $0.5 \%$ & $0.5 \%$ \\
\hline
\end{tabular}

To obtain an equation of motion for the isolated rotor, the oscillations of the blade rows are decoupled by modifying the coefficient matrix $A$. In Equation 8, letting $j=2, n=3$ and $\mathbf{C}_{j k}=0$ for all $k \neq j$ then substituting this $\mathbf{A}$ matrix into Equation 11 results in

$$
\left(\mathbf{S}_{2}+\mathbf{C}_{22}\right) \alpha_{2}=0
$$

for row 2. Thus the oscillations of the blade rows are decoupled, but Equation 24 still retains the effects of unsteady aerodynamic interaction between the blade rows through the coefficient $\mathbf{C}_{22}$. Replacing $\mathbf{C}_{22}$ by the isolated blade row motion-induced moment, $\hat{\mathbf{C}}_{2}$, results in an equation for the isolated rotor:

$$
\left(\mathbf{S}_{2}+\hat{\mathbf{C}}_{2}\right) \boldsymbol{\alpha}_{2}=0 .
$$

$\gamma_{2}$ is determined by solution of

$$
\mathbf{S}_{2}+\hat{\mathbf{C}}_{2}=0 \text {, }
$$

then the oscillation frequency is found from $i \omega_{2} / \omega_{u 2}=i / \sqrt{\gamma_{2}}$. Solutions obtained for the isolated blade row response (NC solution) for $15^{\circ}$ increments in $\beta_{2}$ are shown in Figure 5 ; the corresponding value of $\beta_{2}$ in degrees is given adjacent to each point. The rotor is stable for all $\beta_{2}$

$\left(\operatorname{Re}\left(i \omega_{2} / \omega_{u 2}\right)<0\right)$. Subresonant values of the interblade phase angle $\left(\beta_{2}<-20.3^{\circ}\right.$ and $\left.\beta_{2}>146.4^{\circ}\right)$ are the most stable and form a relatively tight group of points near $\operatorname{Re}\left(i \omega_{2} / \omega_{\nu 2}\right)=-0.12$. Except for $\beta_{2}=150^{\circ}$, the superresonant points form another group which is much closer to the neutral stability line. In particular, the points $60^{\circ} \leq \bar{\beta}_{2} \leq 120^{\circ}$ are relatively close to being unstable.

When aerodynamic coupling is taken into account, but the neighboring blade rows are rigid ( $\mathrm{AC}$ solution),
Equation 24 shows that the rotor frequency-of-oscillation must satisfy

$$
\mathrm{S}_{2}+\mathrm{C}_{22}=0 \text {. }
$$

However, for subresonant values of $\beta_{2}$, all of the acoustic waves decay with axial distance and are therefore neglected in the analysis. Thus in the subresonant region the solutions to Equation 27 will not differ from those to Equation 26. The solutions for the superresonant interblade phase angles have changed, Figure 6 , and some of the roots indicate flutter - the points $75^{\circ} \leq \beta_{2} \leq 135^{\circ}$ have all shifted right far enough that $\operatorname{Re}\left(i \omega_{2} / \omega_{42}\right)>0$.

Solutions to Equation 11 without any simplification (aerodynamic coupling with flexible neighboring blade rows, the DC solution) are shown in Figure 7. About 5 minutes of computing time on a 80486 personal computer was required to obtain these solutions. Now that all of the blade rows are allowed to oscillate, there are three roots at each superresonant interblade phase angle. Where two roots are difficult to distinguish from one another, '(2)' is placed next to the value of $\beta_{2}$. Two distinct groups of roots are shown in the figure - one group, with $\operatorname{Im}\left(i \omega_{2} / \omega_{u 2}\right) \approx 1$, stretches horizontally across the plot while the second group stretches vertically just to the stable side of the neutral stability line. The first group is replotted in Figure 8 along with the $\mathrm{AC}$ solution first shown in Figure 6 . These two solutions are effectively identical, implying that the dynamics of the stator blades have a negligible effect on the rotor stability. Upon inspection of the coefficient matrix $\mathbf{A}$ for this configuration, it was found that the off-diagonal terms were small relative to the diagonal terms, thus the oscillations of the blade rows were effectively decoupled due to weak unsteady aerodynamic interaction. Due to the decoupling, the remaining solutions - the vertical group of roots in Figure 7 - appear to be AC solutions for the stability of the two stators. But the solutions for the stators are nearly identical despite their differing locations in the configuration, which indicates that unsteady aerodynamic coupling effects on them are also negligible. The frequencies of these solutions vary with interblade phase angle because they are presented in terms of the rotor frequency - the stators are oscillating near their natural frequencies, but due to the motion of the rotor, the frequency in the rotor frame varies with the interblade phase angle or tangential wavenumber (Appendix B).

The rotor stability will vary with spacing between it and the upstream stator, $\delta$. Sample results for the present configuration are shown in Figure 9; the positions of the stators are fixed while the rotor position between them is varied between $\delta / c=0$ (rotor leading edge coincident with upstream stator trailing edge) and $\delta / \mathrm{c}=1$ (rotor trailing edge coincident with downstream stator leading edge). Relative 
to the isolated rotor solution, the unsteady aerodynamic coupling effects are generally destabilizing, but for $\delta / c>0.68$, a slight stabilizing effect occurs. The rotor is unstable for $\delta / \subset<0.31$. These results suggest that blade row stability may be enhanced by variation of the blade row spacing.

The stability of a second configuration was also analyzed; configuration II, Table 2, consists of an unstaggered stator between two identical rotor blade rows. The blade row spacing was chosen so that a $1 / 2$ chord axial gap separates the stator from each of the neighboring rotors. For the stator geometry, mean flow conditions and reduced frequency $\bar{\omega}_{2}=1$, the resonant interblade phase angles are $\pm 19.2^{\circ}$, so the superresonant region is relatively small. Figure 10 shows the NC solution for the stator along with the AC solution. The stator is stable in both cases although aerodynamic interaction makes it less stable for some $\beta_{2}$. However, if the rotors are allowed to vibrate, there are dramatic changes in the solution. From Figure 11, the DC solution has two unstable values of $\beta_{2}, 15^{\circ}$ and $10^{\circ}$, and introduces a number of other roots which are quite different from those for the rigid rotors solutions. The dynamics of the neighboring blade rows, which were negligible for configuration $I$, play a crucial role in the stability. of configuration II.

Table 2 Blade row configuration II

\begin{tabular}{|l|c|c|c|}
\hline Blade row index $k$ & $k$ Rotor 1 & Stator & Rotor 2 \\
\hline Chord, $c$ & 1 & 2 & 3 \\
\hline Solidity, $c s$ & 1 & 1 & 1 \\
\hline Stagger angle $($ deg.) & 60 & 0 & 60 \\
\hline Relative Mach no, $M$ & 0.8 & 0.4 & 0.8 \\
\hline Blade Mach no, $U / \sigma$ & -0.693 & 0 & -0.693 \\
\hline Elastic axis / chord & 0.5 & 0.5 & 0.5 \\
\hline Radius of gyration & 0.4 & 0.4 & 0.4 \\
\hline Mass ratio.. & 200 & 200 & 200 \\
\hline Natural freq., $\omega_{\nu} / \omega_{u}$ stator & 1 & 1 & 1 \\
\hline Damping coefficient & $0.5 \%$ & $0.5 \%$ & $0.5 \%$ \\
\hline
\end{tabular}

\subsection{Forced response}

To investigate the effects of unsteady aerodynamic blade row interaction on forced response, calculations were made for the first harmonic response of the stator in configuration $\Pi$ due to the wakes of the upstream blade row. The first harmonic of the wake is assumed to have unity amplitude. The stator reduced frequency is $\bar{\omega}_{2}=16.32$. In contrast to the stability solution procedure, where ratio of the oscillation frequency to the undamped natural frequency was the solution, the oscillation frequency is now fixed and the undamped natural frequency will be varied in order to see how the response varies in the vicinity of resonance.

The forced response of the configuration II stator is shown in Figure 12 as a function of $\omega_{2} / \omega_{y 2}$, the ratio of the stator excitation frequency to the stator undamped natural frequency. The response parameter is the ratio of the oscillation amplitude with unsteady aerodynamic coupling effects to the oscillation amplitude for the isolated stator. At resonance, the AC response is nearly $45 \%$ greater than the $\mathrm{NC}$ response. Away from resonance, the $\mathrm{AC}$ response is still about $20 \%$ greater than the NC response. For this configuration, the DC response was essentially identical to the AC response - the oscillations of the rotors did not affect the stator.

A brief parametric study of the effects of blade row spacing and rotor solidity on the stator forced response was made. Configuration II was used for this study along with two configurations identical to it except for changes in the rotor solidities. The gap between the stator and the upstream rotor was varied between 0 and 1 chord; with a gap of one chord, the stator trailing edge was coincident with the leading edge of the downstream rotor. As shown in Figure 13, the response of the stator can vary greatly with these parameters. With the higher solidity rotors ( $\left.(c / s)_{1}=(c / s)_{3}=1.55\right)$, the stator response amplitude varies between exceeding the isolated stator response by nearly $60 \%$ to being $15 \%$ less than the isolated stator response. In contrast, with the lower solidity rotors $\left((c / s)_{1}=(c / s)_{3}\right.$ $=1.45$ ), the stator response varies little with axial gap, staying at about $13 \%$ greater than the isolated stator response. The configuration II solution falls between these two in both maximum response and fluctuation of the response with blade row spacing. Although it might be tempting to draw some conclusions from the trends of these results, the very limited nature of this study, not to mention the complexity of cascade unsteady aerodynamics, prohibit this.

\section{Summary and Conclusion}

The effects of blade row unsteady aerodynamic interaction on flutter and forced response have been investigated. A model for interaction between any number of blade rows was developed which relies upon unsteady aerodynamic coefficients obtained from isolated blade row analyses. Using influence coefficients which express the unsteady forces on one blade row due to the motion of another, a structural model was obtained which accounts for coupling of the vibratory responses of multiple blade rows, 
or dynamic coupling between the blade rows. A special case of this model is obtained when neighbors of the blade row under consideration are assumed to be rigid, but the unsteady aerodynamic coupling between these neighbors is retained.

This analysis technique was applied to two model configurations, each consisting of three blade rows. Stability solutions were found for the middle blade row of each configuration. For the first configuration, aerodynamic coupling had a destabilizing influence relative to the isolated blade row solution - the isolated blade row solution indicated stability but the unsteady aerodynamic coupling solution indicated flutter. The additional effect of dynamic coupling was negligible relative to the aerodynamic coupling effect. In contrast, the dynamic coupling analysis indicated flutter for the second configuration while the unsteady aerodynamic coupling solution and the isolated blade row solution both predicted stability. It is concluded that both unsteady aerodynamic coupling and dynamic coupling between blade rows can have significant effects on blade row stability.

The forced response analysis was applied to one baseline configuration. Significant increases in the response amplitude were found to occur due to unsteady aerodynamic coupling. A parametric study showed that blade row spacing and solidity can have significant effects on the response amplitude.

\section{Acknowledgment}

This research was supported by the Turbomachinery Technology Branch, Propulsion Systems Division of the NASA Lewis Research Center. L. Bober, D. Hoyniak, D. Huff and F. McCaughan provided helpful comments on the content of this paper.

\section{References}

Chen, S.H. and M.H. Williams, "Panel Method for Counter-Rotating Propfans," Journal of Propulsion, Vol. 7, No. 4.

Chiang, H.-W. D. (1988) "Aerodynamic Detuning of a Loaded Cascade in an Incompresible Flow by a Locally Analytical Method," Ph.D. Thesis, Purdue University School of Mechanical Engineering.

Fang, J. and H.M. Atassi (1993) "Compressible Flows with Vortical Disturbances Around a Cascade of Loaded Airfoils," Unsteady Aerodynamics, Aeroacoustics, and Aeroelasticity of Turbomachines and Propellers H.M. Atassi, editor, Springer-Verlag, New York.
Giles, M.B. (1990) "Stator/Rotor Interaction in a Transonic Turbine," Journal of Propulsion, Vol. 6, No. 5, pp. 621-627.

Hall, K.C. and W.S. Clark (1991) "Prediction of Unsteady Aerodynamic Loads in Cascades Using the Linearized Euler Equations," AIAA Paper 91-3378.

Hanson, D.B. (1992) "Unsteady Coupled Cascade Theory Applied to the Rotor/Stator Interaction Noise Problem," DGLR/AIAA Paper 92-02-084.

Jorgensen, P.C.E. and R.V. Chima (1989) "Explicit Runge-Kutta Method for Unsteady Rotor-Stator Interaction," ALAA Joumal, Vol. 27, No. 6, pp. 473-479.

Kaji, S. and K. Okazaki (1970) "Generation of Sound by Rotor-Stator Interaction," Journal of Sound and Vibration, Vol. 13, No. 3, pp. 281-307.

Rai, M.M. (1987) "Navier-Stokes Simulations of Rotor/Stator Interaction Using Patched and Overlaid Grids," Journal of Propulsion, Vol. 3, No. 5, pp. 387-396.

Silverstein, A., S. Katzoff and W.K. Bullivant (1939) "Downwash and Wake Flow Behind Plain and Flapped Airfoils," NACA Technical Report No. 651.

Smith, S.N. (1972) "Discrete Frequency Sound Generation in Axial Flow Turbomachines," Aeronautical Research Council Reports and Memoranda No. 3709.

Verdon, J.M. (1987) "The Unsteady Flow in the Far Field of an Isolated Blade Row," United Technologies Research Center Report R87-957333-1.

Verdon, J.M. (1990) "Linearized Unsteady Aerodynamics for Turbomachinery Aeroelastic Applications," AIAA Paper 90-2355.

Whitehead, D.S. (1987) "Classical Two-Dimensional Methods," AGARD Manual on Aeroelasticity in Axial-Flow Turbomachines, Volume 1. Unsteady Turbomachinery Aerodynamics, edited by M.F. Platzer and F.O. Carta, AGARD-AG-298 Vol. 1.

Williams, M.H., J. Cho and W.N. Dalton (1991) "Unsteady Aerodynamic Analysis of Ducted Fans," Journal of Propulsion, Vol. 7, No. 5. 


\section{Appendix A Linearized theory of cascade wave propagation}

The unsteady aerodynamic blade row interaction analysis presented in this paper depends upon the ability to predict the characteristics of waves traveling between the blade rows, i.e., the amplitudes, phases and wavenumbers of these disturbances must be known. While the wave amplitudes must be determined by a numerical solution for the isolated blade row unsteady aerodynamics (see Whitehead (1987), Verdon (1990)), which is beyond the scope of this discussion, the wavenumbers and hence wave properties such as spatial decay rates may be determined with some analysis.

The flow is assumed to be inviscid, isentropic, two-dimensional and subsonic. In addition, the mean flow is assumed to be uniform; although this assumption may appear questionable, it is consistent with current linearized cascade analyses which assume that regions of uniform flow exist at some distance upstream and downstream of the cascade. Unsteady disturbances are assumed to be small perturbations to the mean flow field, resulting in a system of first order linear partial differential equations for the unknown perturbation quantities. When harmonic solutions which satisfy cascade periodicity are assumed, a system of linear equations is obtained which may be solved for the disturbance wavenumbers.

The mass conservation equation is, for the $\xi, \eta$ coordinate system of Figure 3,

$$
\frac{D \rho}{D t}+\rho\left(\frac{\partial u}{\partial \xi}+\frac{\partial v}{\partial \eta}\right)=0
$$

The momentum equations are

$$
\frac{D u}{D t}=-\frac{1}{\rho} \frac{\partial p}{\partial \xi}
$$

and

$$
\frac{D v}{D t}=-\frac{1}{\rho} \frac{\partial p}{\partial \eta}
$$

$u$ is the $\xi$ component of velocity, $v$ is the $\eta$ component of velocity, $\rho$ is the density and

$$
\frac{D}{D t} \equiv \frac{\partial}{\partial t}+u \frac{\partial}{\partial \xi}+v \frac{\partial}{\partial \eta}
$$

Using the subscript 0 to denote the constant mean values and $\mathbf{l}$ for the perturbations,

$$
\rho=\rho_{0}+\rho_{1}, u=u_{0}+u_{1}, \ldots
$$

are substituted into the governing conservation equations. Once the equations are expanded and products of perturbations are neglected, a system of linear partial differential equations results with the perturbation quantities as the dependent variables. Conservation of mass becomes

$$
\frac{D_{0 \rho_{1}}}{D t}+p_{0}\left(\frac{\partial u_{1}}{\partial \xi}+\frac{\partial v_{1}}{\partial \eta}\right)=0
$$

The momentum equations become

$$
\frac{D_{0} u_{1}}{D t}=-\frac{1}{\rho_{0}} \frac{\partial p_{1}}{\partial \xi}
$$

and

$$
\frac{D_{0} v_{1}}{D t}=-\frac{1}{\rho_{0}} \frac{\partial p_{1}}{\partial \eta}
$$

with the operator

$$
\frac{D_{0}}{D t} \equiv \frac{\partial}{\partial t}+u_{0} \frac{\partial}{\partial \xi}+v_{0} \frac{\partial}{\partial \eta}
$$

To close the system of equations, the speed of sound for isentropic flow of a calorically perfect gas, $a$, is introduced.

$$
a^{2}=\left(\frac{\partial p}{\partial \rho}\right)_{s}=\frac{d p}{d \rho}
$$

Expanding Equations $A-4$ through $A-6$ and using $A-7$ to replace derivatives of $\rho_{1}$ with derivatives of $p_{1}$ gives

$$
\frac{\partial \mathbf{q}}{\partial t}+\mathbf{A} \frac{\partial \mathbf{q}}{\partial \xi}+\mathbf{B} \frac{\partial \mathbf{q}}{\partial \eta}=0
$$

where

$$
\mathbf{q}=\left(p_{1}, u_{1}, v_{1}\right)^{T}
$$

$$
\mathbf{A}=\left(\begin{array}{ccc}
u_{0} & \rho_{0} a^{2} & 0 \\
1 / \rho_{0} & u_{0} & 0 \\
0 & 0 & u_{0}
\end{array}\right)
$$

and

$$
\mathbf{B}=\left(\begin{array}{ccc}
v_{0} & 0 & \rho_{0} a^{2} \\
0 & v_{0} & 0 \\
1 / \rho_{0} & 0 & v_{0}
\end{array}\right)
$$

The dependent variables are assumed to depend harmonically on the spatial position and the time. Thus the pressure perturbation is expressed as

$$
p_{1}=\overline{p_{1}} e^{i(\omega t+\sqrt{5}+m \eta)}
$$

where $\overline{p_{1}}$ is the pressure disturbance amplitude, $i=\sqrt{-1}$ and $l$ and $m$ are the axial and tangential wave numbers, respectively. Analogous relations are used for $u_{1}$ and $v_{1}$. 
Substituting the perturbation expressions into Equation A-8, a system of linear algebraic equations is obtained:

$$
\left(\begin{array}{ccc}
\omega+u_{0} l+v_{0} m & a^{2} l \rho_{0} & a^{2} m \rho_{0} \\
l / \rho_{0} & \omega+u_{0} l+v_{0} m & \\
m / \rho_{0} & & \omega+u_{0} l+v_{0} m
\end{array}\right)\left(\begin{array}{c}
\overline{p_{1}} \\
\overline{u_{1}} \\
\overline{\nu_{1}}
\end{array}\right)=0 \text {. (A-9) }
$$

To obtain a non-trivial solution to this linear system, the determinant of the coefficient matrix in Equation A-9 is set equal to 0 :

$\left(\omega+u_{0} l+v_{0} m\right)\left[\left(\omega+u_{0} l+v_{0} m\right)^{2}-a^{2}\left(l^{2}+m^{2}\right)\right]=0$.

The two solutions to Equation A-10,

$$
\omega+u_{0} l+v_{0} m=0
$$

and

$$
\left(\omega+u_{0} l+v_{0} m\right)^{2}=a^{2}\left(l^{2}+m^{2}\right)
$$

encompass different physical phenomena.

From the momentum equations, relations between pressure and velocity fluctuations are obtained:

$$
\begin{aligned}
\overline{p_{1}} & =-\frac{\rho_{0}}{l}\left(\omega+u_{0} l+v_{0} m\right) \overline{u_{1}} \\
& =-\frac{\rho_{0}}{m}\left(\omega+u_{0} l+v_{0} m\right) \overline{v_{1}} .
\end{aligned}
$$

Hence for the solution of Equation A-10 obtained specified by Equation $A-11$, the pressure fluctuations are, from Equation A-13, zero, but the vorticity is

$$
\begin{aligned}
\Psi_{1} & =\frac{\partial v_{1}}{\partial \xi}-\frac{\partial u_{1}}{\partial \eta} \\
& =i\left(l \overline{v_{1}}-m \overline{u_{1}}\right) e^{i(\omega t+I \xi+m \eta)} .
\end{aligned}
$$

The phase velocity of this disturbance is determined by tracking a constant disturbance phase. In this reference frame, the phase does not change with time:

$$
\frac{d}{d t}(\omega t+I \xi+m \eta)=0
$$

or

$$
\omega+l \frac{d \xi}{d t}+m \frac{d \eta}{d t}=0
$$

Combining Equations A-15 and A-11 to eliminate $\omega$ results in

$$
l \frac{d \xi}{d t}+m \frac{d \eta}{d t}=u_{0} l+v_{0} m .
$$

Thus the $\xi$ and $\eta$ components of the phase velocity are equal to the mean flow velocity components $u_{0}$ and $v_{0}$. This solution corresponds to convection of vorticity by the mean flow with no associated pressure fluctuations.
For nonzero pressure disturbances, Equation A-13 implies $\omega+u_{0} l+v_{0} m \neq 0$ which is true of the second solution, Equation A-12. From Equations A-17, $m \overline{u_{1}}=l \overline{v_{1}}$. Substituting this into Equation A-14, it follows that the vorticity is zero; the second solution therefore corresponds to irrotational pressure perturbations.

Equation A-12 may be solved for the axial wave number:

$$
l=\frac{u_{0}\left(\omega+v_{0} m\right) \pm a \sqrt{\left(\omega+v_{0} m\right)^{2}-\left(a^{2}-u_{0}^{2}\right) m^{2}}}{a^{2}-u_{0}^{2}} .
$$

The tangential wavenumber must satisfy cascade periodicity, and therefore be compatible with the interblade phase angle $\beta$. Hence

$$
m s=\beta+2 \pi r, \quad r=0, \pm 1, \pm 2, \ldots
$$

where $s$ is the cascade spacing. Because $m$ depends upon real quantities, it is also real.

The nature of acoustic wave propagation depends upon l. From Equation A-17, if $\left(\omega+v_{0} m\right)^{2}-\left(a^{2}-u_{0}^{2}\right) m^{2}<0$, then $l$ will be complex. Let $l=l^{R e}+i l^{I m}$ so that

$$
\begin{aligned}
& p_{1}=\overline{p_{1}} e^{i\left(\omega t+\left(l^{\left.\mu_{1}+i l^{m}\right)}\right)_{j}+m \eta\right)} \\
& =\overline{p_{1}} e^{-l^{l m \xi}} e^{i\left(\omega t+l^{n} \xi+m\right)}
\end{aligned}
$$

which indicates that the wave either grows or decays exponentially with axial distance. The amplifying wave solution is commonly disallowed on the basis that it is not physically acceptable (Fang and Atassi (1993), Verdon (1987)), leaving only the decaying wave solution. If $\left(\omega+v_{0} m\right)^{2}-\left(a^{2}-u_{0}^{2}\right) m^{2}>0, l$ is real and the acoustic waves will propagate unattenuated, one upstream (for the $(-)$ sign in Equation A-17) and one downstream (for the + sign in Equation A-17) - see Whitehead (1987). At the acoustic resonance or cutoff condition, $\left(\omega+v_{0} m\right)^{2}=\left(a^{2}-u_{0}^{2}\right) m^{2}$. Solving for $m_{c}$, the tangential wavenumber at cutoff,

$$
m_{c} \equiv m_{\text {cutoff }}=\oplus\left(\frac{v_{0} \pm \sqrt{a^{2}-u_{0}^{2}}}{a^{2}-u_{0}^{2}-v_{0}^{2}}\right)
$$

or

$$
m_{c}=\frac{\omega}{a}\left(\frac{M_{\eta} \pm \sqrt{1-M_{\xi}^{2}}}{1-M^{2}}\right) .
$$




\section{Appendix B Frequency relations and scattering effects}

Let a disturbance be described as

$$
B=\bar{B} \exp \left[i\left(\omega_{1} t+l \xi_{1}+m \eta_{1}\right)\right]
$$

in a $\xi_{1}, \eta_{1}$ reference frame moving at velocity $v_{\xi_{1}}=0, v_{\eta_{1}}=U_{1}$ with respect to a fixed $\xi, \eta$ coordinate system. Let a second reference frame with coordinates $\xi_{2}, \eta_{2}$ have velocity $v_{\xi_{2}}=0, v_{\eta_{2}}=U_{2}$ with respect to the $\xi, \eta$ coordinates. Thus

$$
\xi_{j}=\xi
$$

and

$$
\eta=\eta_{j}+U_{j} t
$$

for $j=1,2$. Using Equation B-3 twice, first for $j=1$ and then for $j=2$, then equating the results gives

$$
\eta_{1}=\eta_{2}+\left(U_{2}-U_{1}\right) t
$$

Substituting $B-2$ and $B-4$ into $B-1$ yields

$$
\begin{aligned}
B & =\bar{B} \exp \left\{i\left[\omega_{1} t+l \xi_{2}+m\left(\eta_{2}+\left(U_{2}-U_{1}\right) t\right)\right]\right\} \\
& =\bar{B} \exp \left\{i\left[\left(\omega_{1}+m\left(U_{2}-U_{1}\right)\right) t+l \xi_{2}+m \eta_{2}\right]\right\} .
\end{aligned}
$$

B-6 implies that $\omega_{2}$, the frequency in the second reference frame, is

$$
\omega_{2}=\omega_{1}+m\left(U_{2}-U_{1}\right) .
$$

Thus the frequencies generally differ due to relative tangential motion between blade rows, with the frequency shift depending upon the tangential wavenumber $m$ in addition to the velocity difference.

The appearance of $m$ in equation B-6 has important consequences when scattering effects are considered. As an example, consider the first harmonic forced response of a stator (row 2) by the wakes from an upstream rotor (row 1). For the first harmonic of the wake, the tangential wavenumber is

$$
m=\frac{2 \pi}{s_{1}}
$$

where $s_{1}$ is the rotor blade spacing. Since $U_{2}=0$, the excitation frequency of the stator is, from Equation B-6,

$$
\omega_{2}=\omega_{1}-m U_{1} .
$$

But the wake disturbance frequency is zero in the rotor frame so $\omega_{1}=0$ and

$$
\omega_{2}=-m U_{1}=-\frac{2 \pi}{s_{1}} U_{1}
$$

Waves created by interaction of the gust with the stator must satisfy the interblade phase angle imposed by the gust, i.e.,

$$
m^{q} s_{2}=\beta_{2}+2 \pi q, \quad q= \pm 1, \pm 2, \ldots
$$

where $m^{q}$ is the tangential wavenumber of a scattered wave and

$$
\beta_{2}=m s_{2}=2 \pi \frac{s_{2}}{s_{1}} .
$$

Substituting B-11 into B-10 and rearranging yields

$$
m^{q}=2 \pi\left(\frac{1}{s_{1}}+\frac{q}{s_{2}}\right)
$$

Using Equations B-8 and B-12, any of these scattered waves incident on the rotor will be at frequencies (in the rotor frame)

$$
\begin{aligned}
\omega_{1}^{q} & =\omega_{2}+m^{q} U_{1} \\
& =\left(m^{q}-m\right) U_{1} \\
& =\frac{2 \pi q}{s_{2}} U_{1} .
\end{aligned}
$$

The rotor will scatter the incident waves to wavenumbers

$$
\begin{aligned}
m^{q, r} & =m^{q}+\frac{2 \pi r}{s_{1}} \quad r=0, \pm 1, \pm 2, \ldots \\
& =2 \pi\left(\frac{1+r}{s_{1}}+\frac{q}{s_{2}}\right)
\end{aligned}
$$

at frequencies according to Equation B-13. Finally, the $m^{q, r}$ waves coming from the rotor will excite the stator at frequencies

$$
\begin{aligned}
\omega_{2}^{r} & =\omega_{1}^{q}-m^{q, r} U_{1} \\
& =-\frac{2 \pi(1+r)}{s_{1}} U_{1} .
\end{aligned}
$$

The point of this example is that scattering at the rotor affects the frequencies seen by the stator and vice versa. As shown by Equation B-15, waves scattered at the rotor (i.e., $r \neq 0$ ) return to the stator at a frequency which differs from $\omega_{2}$, the frequency of interest, as given by Equation B-9. Upon further scattering, some of wave energy will return to the frequency $\omega_{2}$ - this effect is neglected in this paper. 

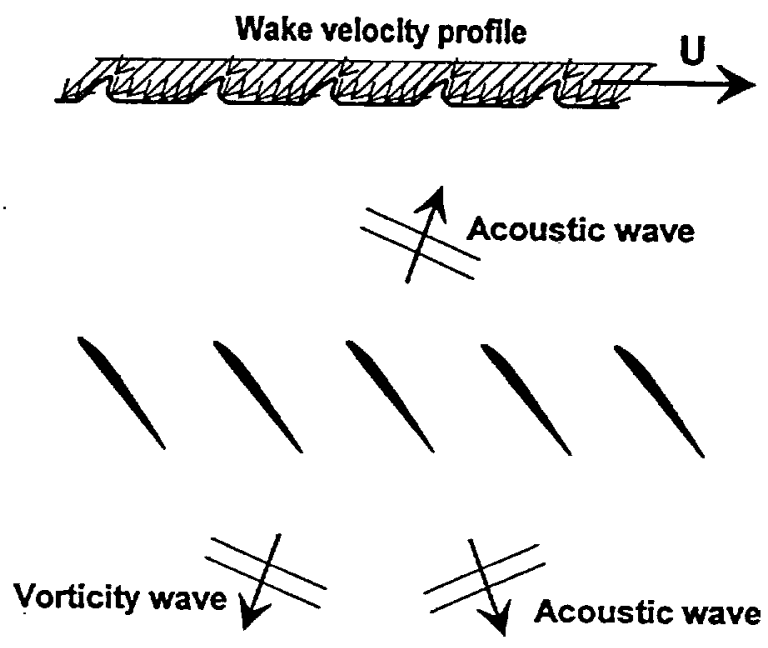

Figure 1 Wave propagation from isolated blade row due to forced response

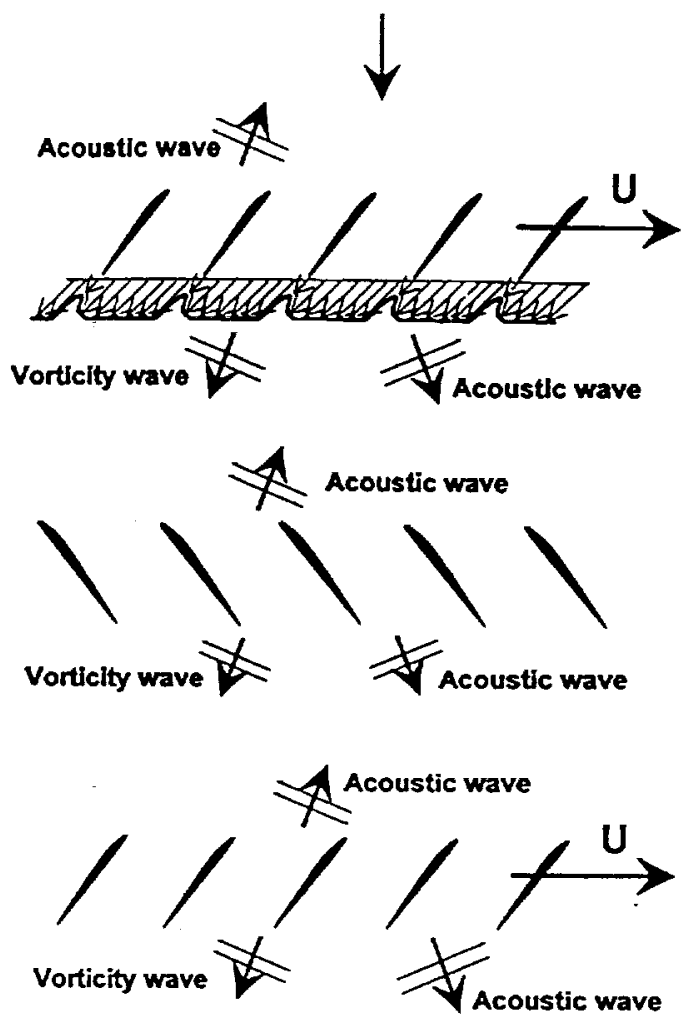

Figure 2 Wave propagation due to forced response of blade row in multistage turbomachine

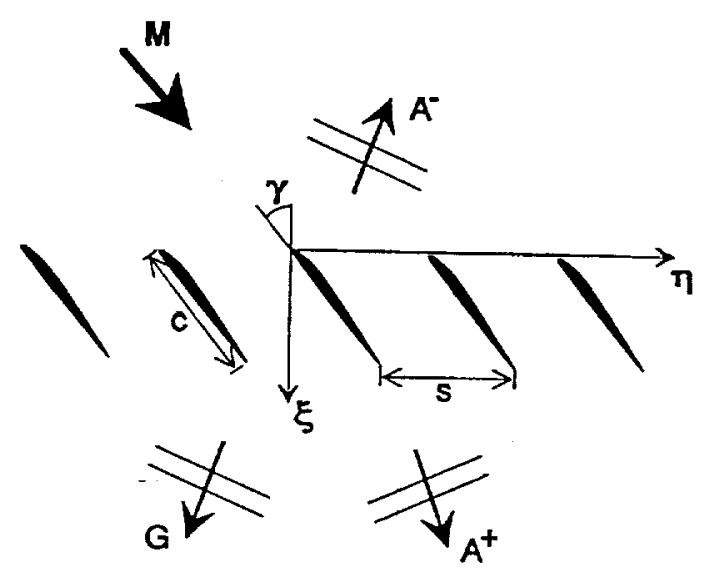

Figure 3 Cascade nomenclature 


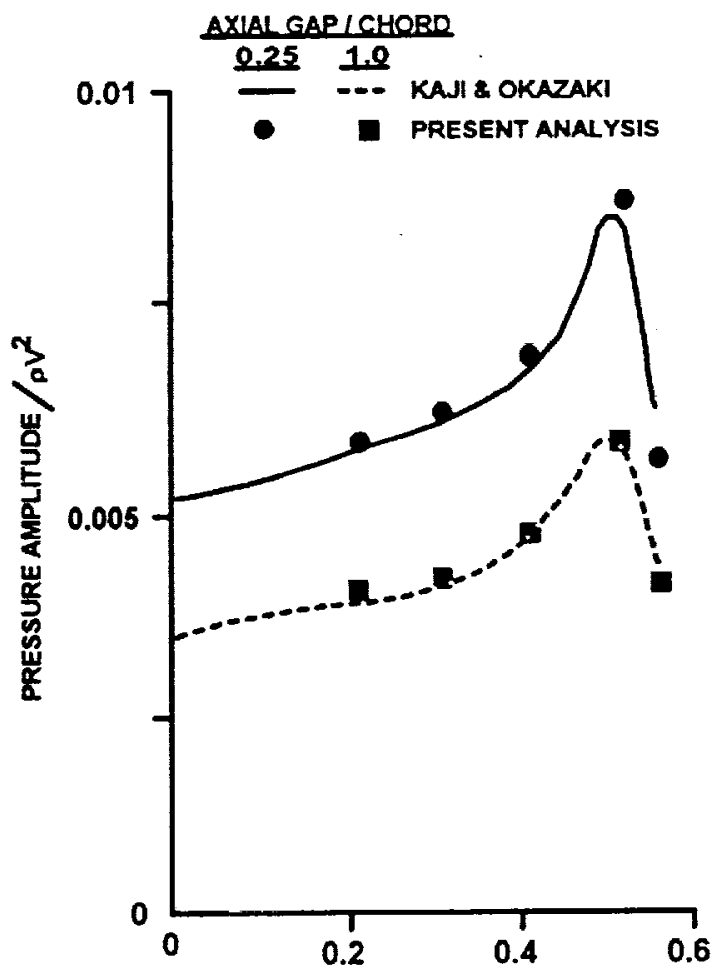

(a) Upstream-running acoustic wave

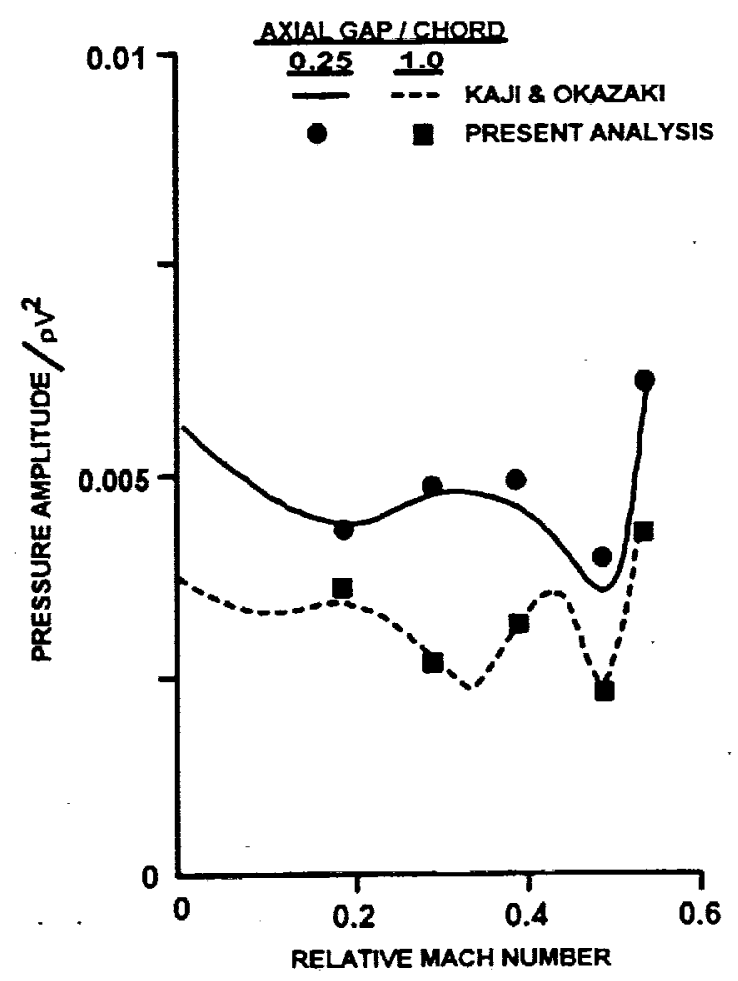

(b) Downstream-running acoustic wave

Figure 4 Rotor-stator interaction noise 


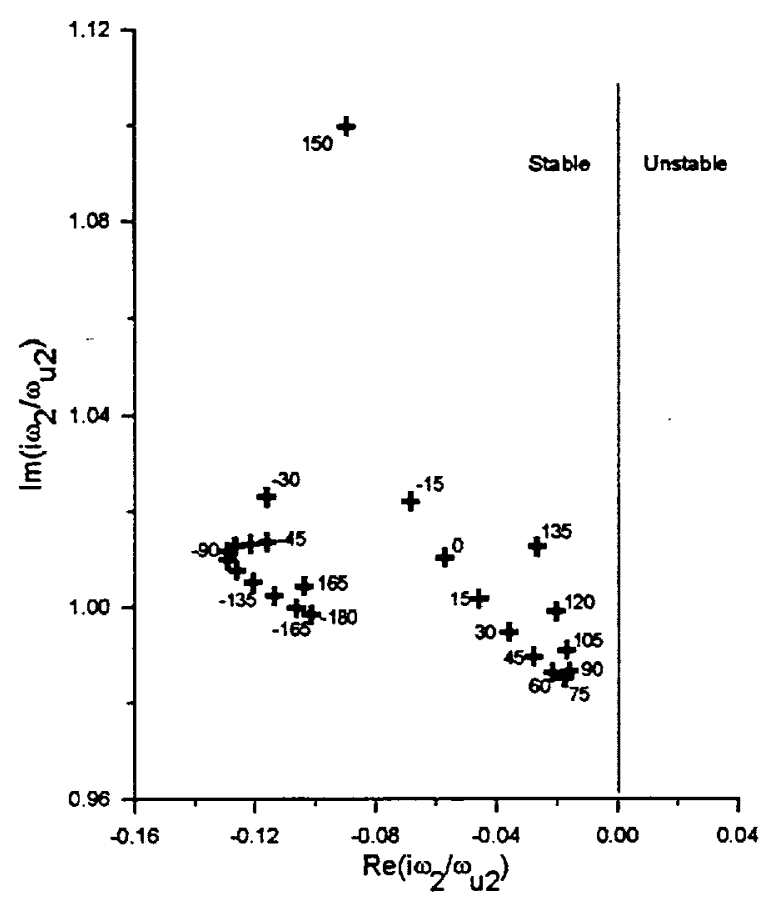

Figure 5 Configuration I isolated rotor stability (interblade phase angle adjacent to symbol)

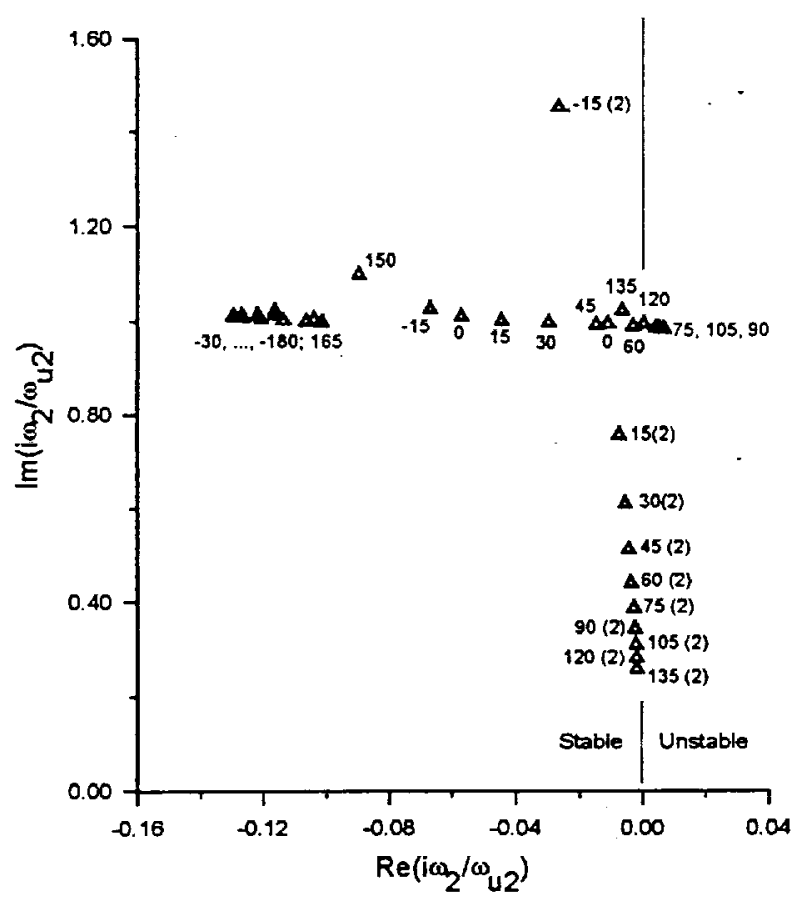

Figure 7 Configuration I stability, dynamic coupling (DC) solution

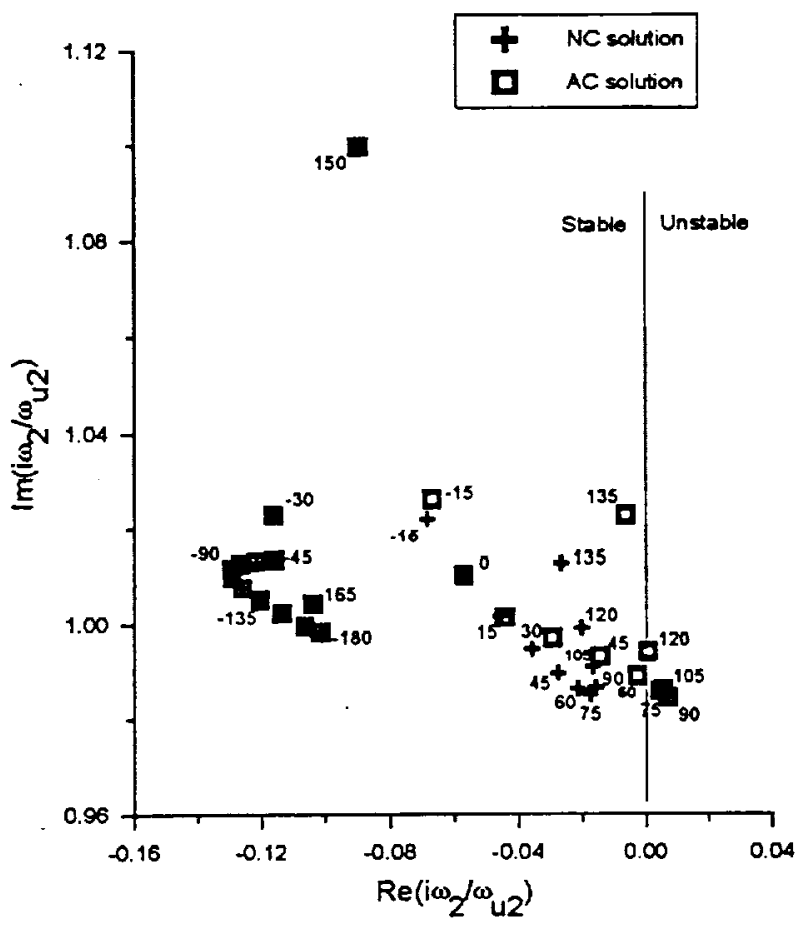

Figure 6 Configuration I rotor stability: comparison of unsteady aerodynamic coupling $(A C)$ and no coupling (NC) solutions

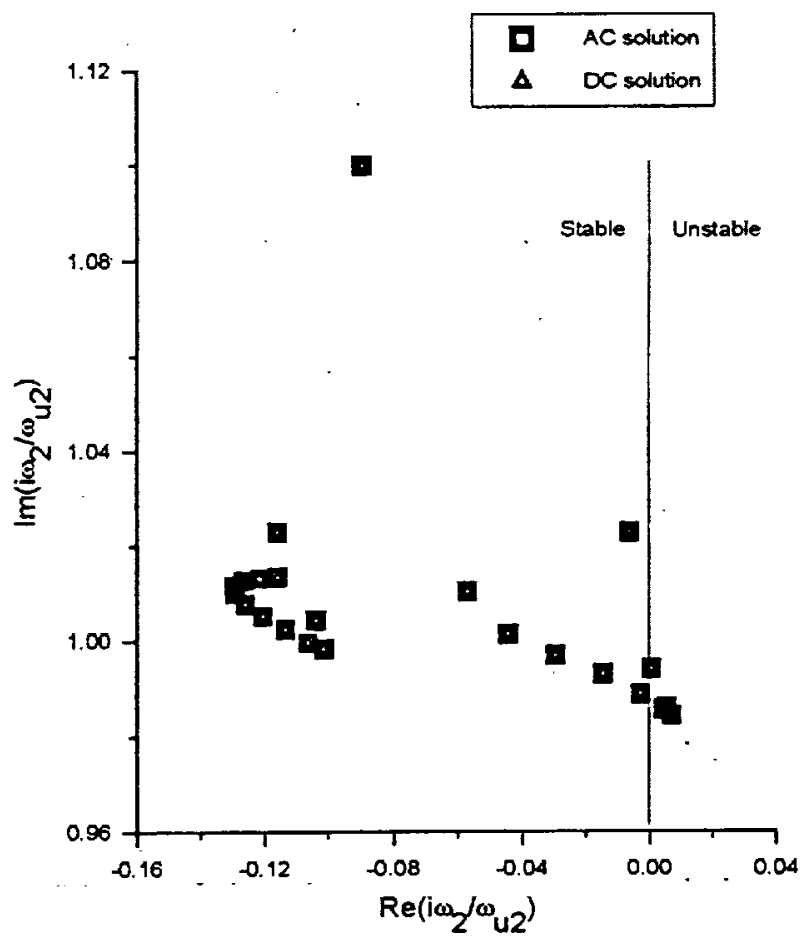

Figure 8 Configuration I stability: comparison of unsteady aerodynamic coupling (AC) solution with dynamic coupling (DC) solution 


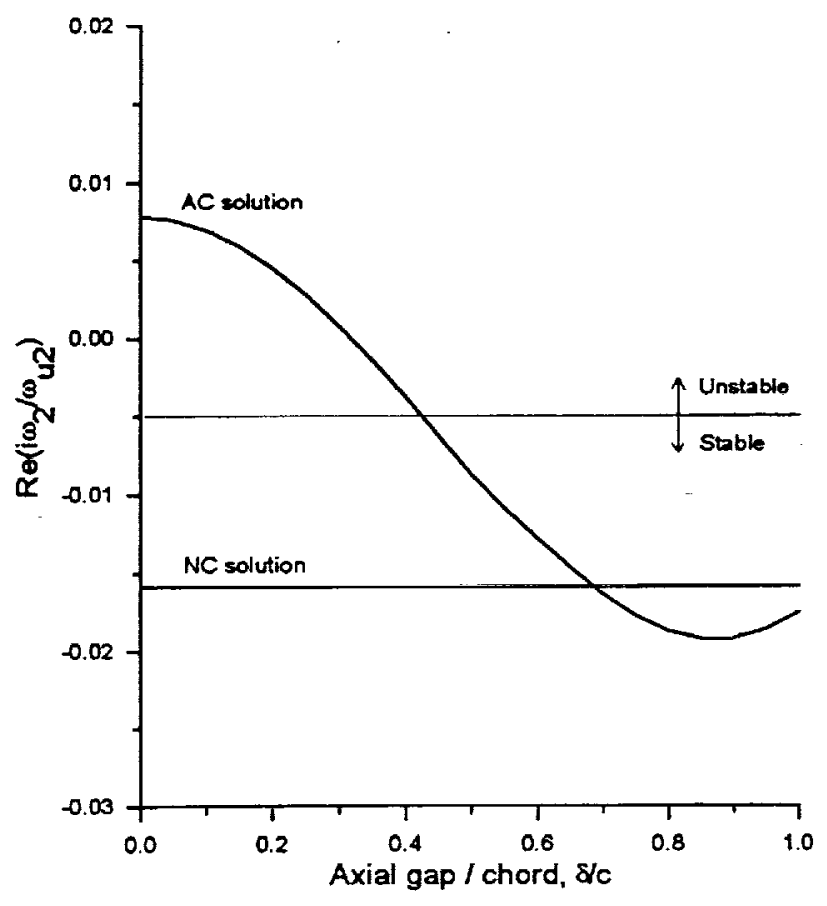

Figure 9 Effect of axial gap on least stable solution, configuration I

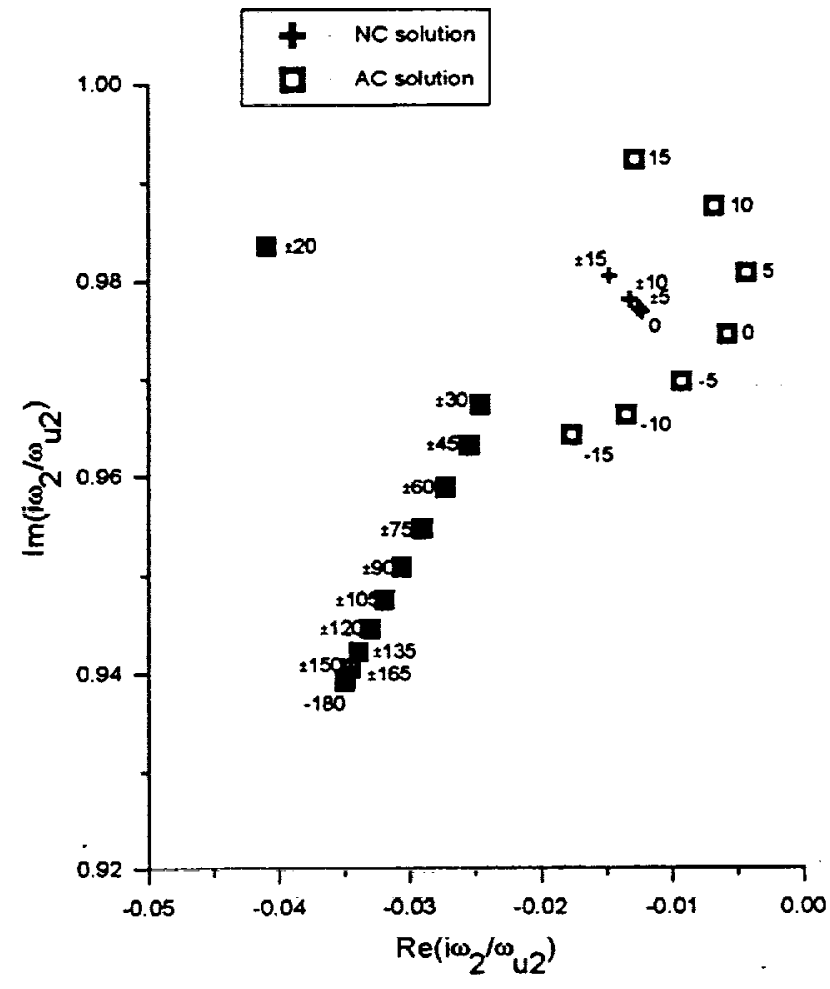

Figure 10 Configuration II stator stability: unsteady aerodynamic coupling (AC) solution vs. no coupling (NC) solution

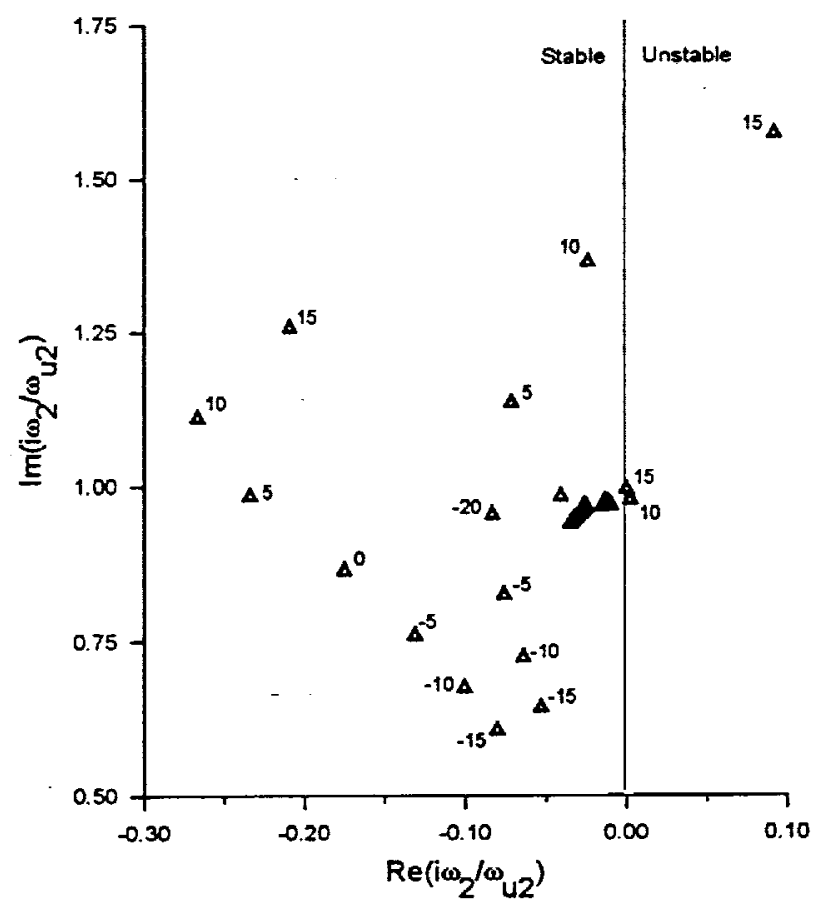

Figure 11 Configuration II stability: dynamic coupling solution 


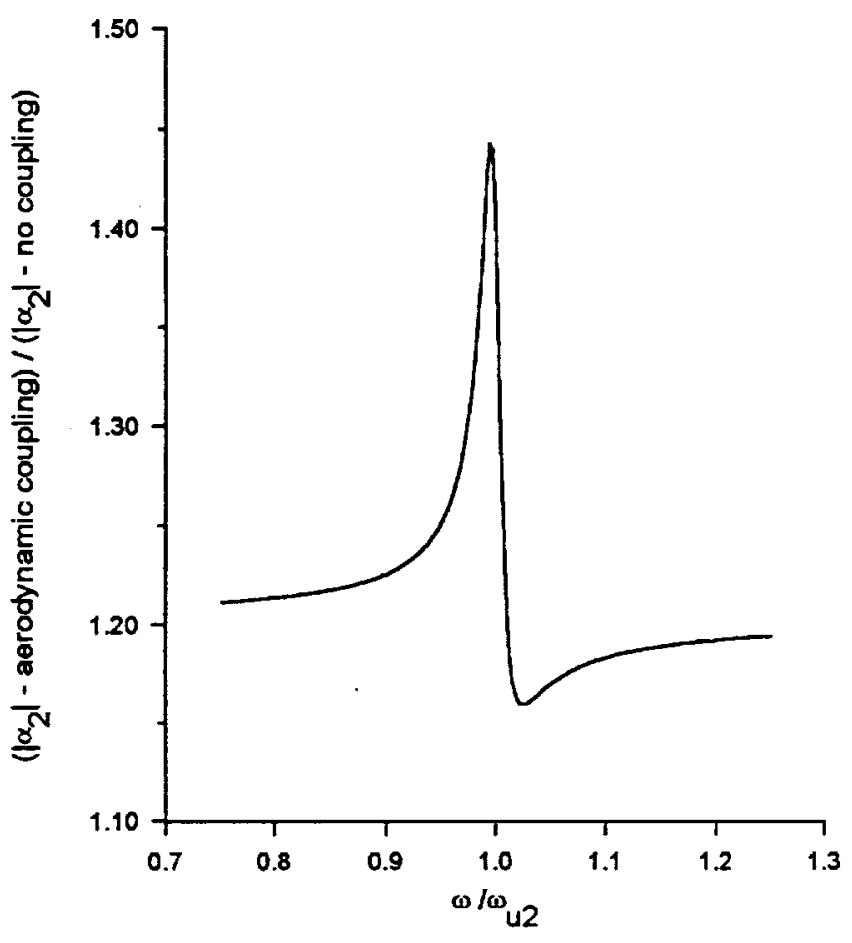

Figure 12 Forced response of configuration II stator vs. frequency

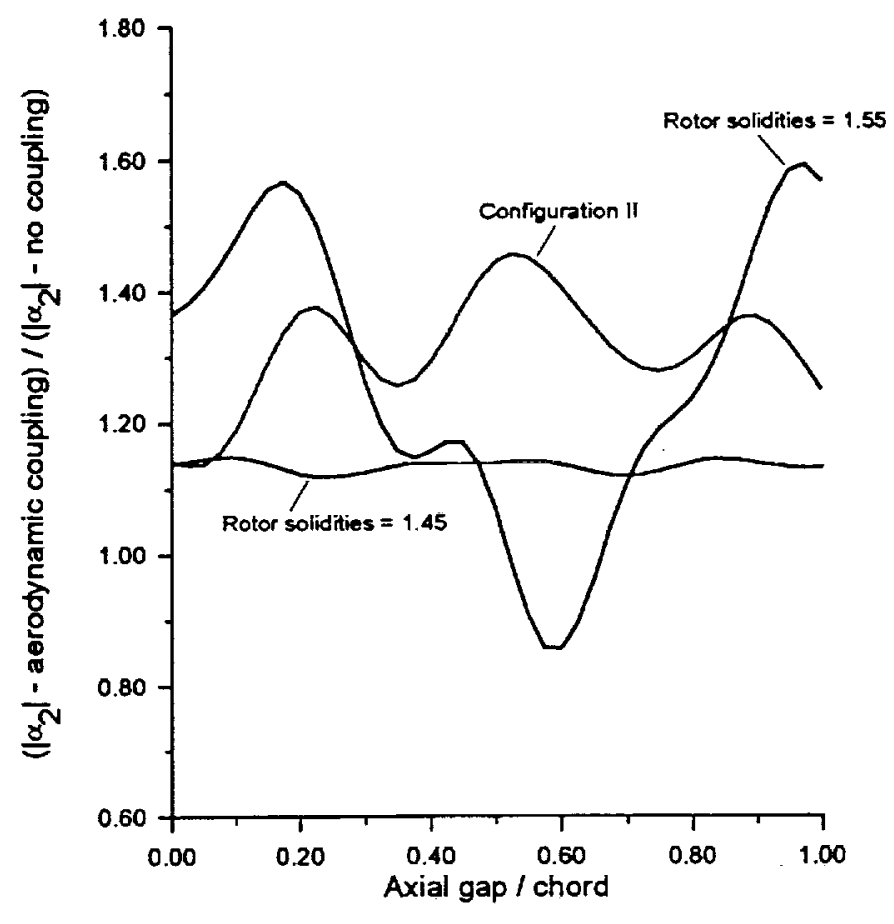

Figure 13 Effect of blade row spacing and rotor solidities on peak stator forced response 
Public reporting burden for this collection of information is estimated to average 1 hour per response, including the time for reviewing instructions, searching existing data sources, gatherling and maintaining the data needed, and completing and revlewing the collection of intormation. Send comments regarding this burden estimale or any other aspect of this collection of information, including suggestions for redueing this burden, to Washington Headquarters Services, Directorate for Information Operations and Reports, 1215 Jeffer
Davis Highway, Suite 1204, Arlington, VA 22202-4302, and to the Office of Management and Budget, Paperwork Reduction Project (0704-0188), Washington, DC 20503.

\begin{tabular}{|l|l|r|}
\hline 1. AGENCY USE ONLY (Leave blank) & $\begin{array}{r}\text { 2. REPOAT DATE } \\
\text { December } 1993\end{array}$ & $\begin{array}{r}\text { 3. REPORT TYPE AND DATES COVERED } \\
\text { Technical Memorandum }\end{array}$ \\
\hline
\end{tabular}

4. TITLE AND SUBTITLE

Blade Row Interaction Effects on Flutter and Forced Response
5. FUNDING NUMBERS

WU-505-62-10

\section{AUTHOR(S)}

Daniel H. Buffum

\section{PERFORMING ORGANIZATION NAME(S) AND ADDRESS(ES)}

National Aeronautics and Space Administration

Lewis Research Center

Cleveland, Ohio 44135-3191

\section{PERFORMING ORGANIZATION} REPORT NUMBER

E-8280

9. SPONSORING/MONITORING AGENCY NAME(S) AND ADDRESS(ES)

National Aeronautics and Space Administration

Washington, D.C. 20546-0001

10. SPONSORINGMONITORING AGENCY REPORT NUMBER

NASA TM-106438

AIAA -93-2084

11. SUPPLEMENTARY NOTES

Prepared for the 29th Joint Propulsion Conference, cosponsored by the AIAA, SAE, ASME, and ASEE, Monterey, California, June 28-July 1, 1993. Responsible person, Daniel H. Buffum, (216) 433-3759.

12a. DISTRIBUTION/AVAILABILITY STATEMENT

12b. DISTRIBUTION CODE

Unclassified - Unlimited

Subject Category 07

13. ABSTRACT (Maximum 200 words)

In the flutter or forced response analysis of a turbomachine blade row, the blade row in question is commonly treated as if it is isolated from the neighboring blade rows. Disturbances created by vibrating blades are then free to propagate away from this blade row without being disturbed. In reality, neighboring blade rows will reflect some portion of this wave energy back toward the vibrating blades, causing additional unsteady forces on them. It is of fundamental importance to determine whether or not these reflected waves can have a significant effect on the aeroelastic stability or forced response of a blade row. Therefore, a procedure to calculate intra-blade-row unsteady aerodynamic interactions has been developed which relies upon results available from isolated blade row unsteady aerodynamic analyses. In addition, an unsteady aerodynamic influence coefficient technique is used to obtain a model for the vibratory response in which the neighboring blade rows are also flexible. The flutter analysis shows that interaction effects can be destabilizing, and the forced response analysis shows that interaction effects can result in a significant increase in the resonant response of a blade row.

\begin{tabular}{|c|c|}
\hline $\begin{array}{c}\text { 14. SUBJECT TERMS } \\
\text { Flutter; Blade row; Acoustic; Rotor-starter }\end{array}$ \\
\hline $\begin{array}{c}\text { 17. SECURITY CLASSIFICATION } \\
\text { OF REPORT } \\
\text { Unclassified }\end{array}$ & $\begin{array}{c}\text { 18. SECURITY CLASSIFICATION } \\
\text { OF THIS PAGE } \\
\text { Unclassified }\end{array}$ \\
\hline
\end{tabular}

\begin{tabular}{|c|c|}
\hline & $\begin{array}{l}\text { 15. NUMBER OF PAGES } \\
19\end{array}$ \\
\hline & $\begin{array}{l}\text { 16. PRICE CODE } \\
\qquad \mathrm{A} 03\end{array}$ \\
\hline $\begin{array}{l}\text { 19. SECURTY CLASSIFICATION } \\
\text { OF ABSTRACT } \\
\text { Unclassified }\end{array}$ & 20. LIMITATION OF ABSTRACT \\
\hline
\end{tabular}

